ni e dalla citazione di oltre 160 voci bibliografiche, la monografia è presentata in chiara veste editoriale.

\section{Mario Crrieli}

Torsten Sjögren and Tage Larsson: Oligophrenia in combination with congenital ichtbyosis and spastic disorders. Acta Psychiatrica et neurologica scandinavica. Supplementum 113, Volumen 32, Einar Munksgaard Ed. Copenhagen 1957.

Gli AA. presentano 28 casi di una nuova sindrome caratterizzata da oligofrenia di basso grado, ittiosi congenita e disordini spastici del tipo della malattia di Little; di tale sindrome non sono stati riscontrati precedenti nella letteratura medica mondiale.

Il quadro clinico è caratterizzato da oligofrenia congenita e stazionaria di basso grado (idiozia o imbecillità), notevole ittiosi congenita stazionaria (che può essere diagnosticata come eritrodemia ittiosiforme) e sintomi spastici piramidali simmetrici congeniti delle estremità. Ed è tanto evidente che la diagnosi può essere fatta sicuramente sulla base del solo esame clinico. Non sono state osservate deviazioni dalla norma in due casi sottoposti ad accu- rati esami biochimici e sierologici. In tre casi è stata diagnosticata la degenerazione dell'epitelio pigmentato della macula e suoi dintorni. L'accurata analisi genetica dei casi e delle loro parentele (circa 2.000 persone) ha dimostrato che in questa sindrome non. si rivela l'influenza dei fattori esogeni ma una causa genetica con ereditarietà monoibrida autosomica recessiva. Infatti tutti i congiunti dei pazienti (eccetto 3) erano nativi della Contea di Västerbotten, nel nord della Svezia, ove è stata rintracciata una zona ristretta del paese con una prevalenza del numero degli eterozigotici. I risultati dell'analisi genealogica e geografica indicano che per la gran maggioranza dei pazienti l'origine della malattia è dovuta ad un'unica mutazione genica - che si presenta nel $0,015 \%$ della popolazione della Contea -; in altri gruppi della popolazione svedese sembra probabile la presenza dello stesso germe aberrante, ma con origini da altre mutazioni, in non più del $0,0005 \%$.

La pregevole monogratia è corredata dagli alberi genealogici di tutti i casi esaminati, con numerose tavole ed illustrazioni; ha una abbondante bibliografia ( 59 voci), ed è presentata nell'usuale ottima veste editoriale.

Mario Cirielli 